\title{
Correlation of Severity of COVID-19 Disease With Gastrointestinal Manifestations and Liver Injury - A North Brooklyn Community Hospital Experience: A Retrospective Cohort Study
}

\author{
Kitson Deane ${ }^{1}$, Ajay Singh ${ }^{2}$, Azza Sarfraz ${ }^{3}$, Zouina Sarfraz ${ }^{4}$, Lyam Ciccone ${ }^{1}$, Beishi Zheng ${ }^{1}$, Arslan \\ Afzal $^{1}$, Gulam Khan ${ }^{5}$, Giovanna Rodriguez ${ }^{6}$, Gul Bahtiyar ${ }^{6}$ \\ 1. Internal Medicine, Woodhull Medical Center, Brooklyn, USA 2. Internal Medicine, Metropolitan Hospital, New York \\ City, USA 3. Pediatrics, Aga Khan University, Karachi, PAK 4. Research and Academic Affairs, Fatima Jinnah Medical \\ University, Lahore, PAK 5. Gastroenterology, Woodhull Medical Center, Brooklyn, USA 6. Endocrinology, Woodhull \\ Medical Center, Brooklyn, USA
}

Corresponding author: Kitson Deane, ktmi_1@hotmail.com

\section{Abstract \\ Introduction}

The primary receptor for SARS-CoV-2 infection, angiotensin-converting enzyme-2 (ACE-2), is expressed in the gastrointestinal tract and liver parenchyma. The involvement of the gastrointestinal tract with severe acute respiratory syndrome coronavirus-2 (SARS-CoV-2) infection has remained unclear. The following study retrospectively reviews gastrointestinal symptoms and liver function tests at the time of hospital admission to identify patient outcomes including prolonged hospital stay, the requirement for intensive care, and all-cause in-hospital 30-day mortality.

\section{Methods}

A retrospective review of patient charts at the Woodhull Medical and Mental Health Center (WMC) was conducted at the time of hospital admission, using a pre-determined selection criterion. All adult patients, both inpatient and outpatient, were included from March 2020 till May 2020. A 95\% confidence interval was used to estimate the odds ratio (OR) for patient outcomes.

\section{Results}

Of the 520 patients, gastrointestinal symptoms including nausea $(\mathrm{OR}=0.375, \mathrm{p}=0.015)$, and nausea and vomiting in combination ( $\mathrm{OR}=0.400, \mathrm{p}=0.016$ ) had an inverse protective relationship with all-cause inhospital 30-day mortality among COVID-19 patients. Gastrointestinal symptoms including diarrhea $(\mathrm{OR}=$ $1.008, \mathrm{p}<0.001)$, and nausea and vomiting $(\mathrm{OR}=1.291, \mathrm{p}=0.043)$ had a mild impact on the length of hospital stay.

Review began 04/11/2021 Review ended 04/15/2021 Published 04/18/2021

\section{(๑) Copyright 2021}

Deane et al. This is an open access article distributed under the terms of the Creative Commons Attribution License CC-BY 4.0., which permits unrestricted use, distribution, and reproduction in any medium, provided the original author and source are credited.

\section{Conclusion}

Elevated liver transaminases including alanine transaminase (ALT) and aspartate transaminase (AST) at the time of hospital admission can predict critical care requirement and all-cause 30-day hospital mortality in patients with COVID-19 infection. Presence of gastrointestinal symptoms is associated with worsened outcomes.

Categories: Gastroenterology, Infectious Disease, Epidemiology/Public Health

Keywords: symptoms, liver, transaminases, prognosis, covid-19, gastrointestinal

\section{Introduction}

Ever since coronavirus disease 2019 (COVID-19) was declared a global health emergency, it has caused over 2.48 million deaths as of February 23, 2021. The respiratory tract manifestations have been commonly observed with fever and cough being reported most frequently [1]. However, recent studies have demonstrated that the gastrointestinal tract and liver may also serve as target organs for severe acute respiratory syndrome coronavirus-2 (SARS-CoV-2) [2]. Such findings are corroborated on the basis that the angiotensin-converting enzyme 2 (ACE-2), the primary receptor for SARS-CoV-2, is presented in the gastrointestinal tract and liver parenchyma [3]. While the impact of SARS-CoV-2 on the gastrointestinal tract and liver remains unclear, COVID-19 patients presenting with digestive symptoms and deranged liver function tests (LFTs) may have a worse prognosis [4].

We conducted a retrospective review of patient data to correlate the presence of digestive symptoms and derangement of LFTs with the duration of hospital stay, the requirement for intensive care, and 30-day 


\section{Cureus}

mortality of COVID-19 patients admitted to Woodhull Medical Center from March 2020 till May 2020.

\section{Materials And Methods}

\section{Study design and population}

A retrospective review of patient charts at the Woodhull Medical and Mental Health Center (WMC) was conducted. All patients aged 18 years and above were included with no restriction to gender, race, or ethnicity. Only pre-existing data, both inpatient and outpatient, was included from March 2020 till May 2020. Data were obtained from patient records, both electronic and paper records, from the WMC. Specific data were reviewed including patient demographics, gastrointestinal symptomatology, laboratory values at the time of hospital admission, progress notes, and procedure notes.

\section{Data collection}

Privacy and security were maintained by password protecting identifiable data and minimizing access to identifiable data. All datasheets were password protected and all paper records were stored in a keyed file drawer in a locked office. Following the acquisition of information, surrogate numbers were given to identify patients for data analysis, and a key was kept in a separate password-protected excel sheet to be searched until after completion of the study at which time it was deleted. The principal and co-investigators reviewed the charts and were the only members to have access to the subjects' data.

\section{Statistical analysis}

The statistical analysis was conducted to assess the role of various factors in patient outcomes. The frequency of gastrointestinal symptoms and elevated LFTs were specified. Only the parameters obtained at the time of hospital admission were used to conduct the statistical analysis. The primary outcome was to determine the 30-day mortality, ICU admission, and hospital stay following COVID-19 diagnosis for enrolled patients. The results of the multivariate analysis were presented in terms of the odds ratio (OR). A difference with a two-sided $\alpha<0.05$ was considered statistically significant. The data analysis was conducted on Statistical Package for Social Sciences (SPSS) version 26.0 (IBM Corp., Armonk, NY).

\section{Results}

A total of 520 patients were reviewed retrospectively who were polymerase chain reactive (PCR) positive for COVID-19. Among these, 60 patients had only nausea, 37 patients had only vomiting, and 63 patients with nausea and vomiting. Only 64 patients had diarrhea as their only gastrointestinal manifestation. A total of 105 patients had a combination of one or more of nausea, vomiting, or diarrhea (Table 1).

\begin{tabular}{|c|c|c|c|c|c|c|}
\hline & patients & $\begin{array}{l}\text { Patients with } \\
\text { nausea }\end{array}$ & $\begin{array}{l}\text { Patients with } \\
\text { vomiting }\end{array}$ & $\begin{array}{l}\text { Patients with nausea and } \\
\text { vomiting }\end{array}$ & $\begin{array}{l}\text { Patients with } \\
\text { diarrhea }\end{array}$ & $\begin{array}{l}\text { Patients with any combination of one or } \\
\text { more of the } 3\end{array}$ \\
\hline $\mathrm{n}$ & 520 & 60 & 37 & 63 & 64 & 105 \\
\hline
\end{tabular}

\section{TABLE 1: Gastrointestinal symptomatology of participants}

Of the 520 patients, 344 patients had deranged international normalized ratio (INR) (Table 2). Nearly all of the patients $(n=488)$ had elevated aspartate transaminase (AST) and alanine transaminase (ALT) in combination (Table 2).

\begin{tabular}{|l|l|l|l|l|}
\hline & All patients & Patients with deranged INR & Patients with elevated AST and ALT & Patients with decreased albumin (<3.5) \\
\hline n 520 & 344 & 488 & 481 \\
\hline
\end{tabular}

TABLE 2: Laboratory findings of participants

INR: International normalized ratio; AST: Aspartate transaminase; ALT: Alanine transaminase. 


\section{Cureus}

$=0.400, \mathrm{p}=0.016)$, had a significant 30-day mortality rate (Table 3). Elevation of ALT $(\mathrm{OR}=1.558, \mathrm{p}=0.038)$, AST $(O R=2.743, p<0.001)$, and in combination $(O R=2.593, p<0.001)$ had a significant impact on the $30-$ day mortality rate (Table 3).

\begin{tabular}{|c|c|c|c|c|c|}
\hline \multirow{2}{*}{ Symptoms } & \multirow{2}{*}{ 30-day mortality rate } & \multirow{2}{*}{ Odds ratio (OR) } & \multicolumn{2}{|c|}{$95 \%$ Confidence Interval } & \multirow{2}{*}{ P-value } \\
\hline & & & Lower & Upper & \\
\hline Nausea & $5.8 \%$ & 0.375 & 0.166 & 0.849 & 0.015 \\
\hline Vomiting & $4.2 \%$ & 0.471 & 0.179 & 1.239 & 0.160 \\
\hline Diarrhea & $9.0 \%$ & 0.630 & 0.325 & 1.22 & 0.222 \\
\hline Nausea and Vomiting & $6.7 \%$ & 0.400 & 0.185 & 0.864 & 0.016 \\
\hline Elevated INR (>1.5) & $17.3 \%$ & 1.824 & 0.941 & 3.536 & 0.072 \\
\hline Elevated Transaminases (AST > 40 and ALT > 45) & $78.2 \%$ & 2.593 & 1.635 & 4.111 & 0.000 \\
\hline AST $>40$ & $33.6 \%$ & 2.743 & 1.739 & 4.327 & 0.000 \\
\hline $\mathrm{ALT}>45$ & $32.9 \%$ & 1.558 & 1.023 & 2.372 & 0.038 \\
\hline Low Albumin $(<3.5)$ & $43.9 \%$ & 1.120 & 0.749 & 1.676 & 0.580 \\
\hline
\end{tabular}

\section{TABLE 3: Predictors of 30-day mortality rate}

INR: International normalized ratio; AST: Aspartate transaminase; ALT: Alanine transaminase.

Diarrhea as a gastrointestinal (GI) manifestation was a significant contributor to prolonged hospital stay lasting $\geqslant 10$ days $(O R=1.008, p<0.001)$ (Table 4$)$. Nausea and vomiting were also associated with prolonged hospital stay lasting $\geqslant 10$ days $(\mathrm{OR}=1.291, \mathrm{p}=0.043)($ Table 4$)$.

\begin{tabular}{|c|c|c|c|c|c|c|}
\hline \multirow{2}{*}{ Symptoms } & \multicolumn{2}{|c|}{ Hospital Stay } & \multirow{2}{*}{ OR } & \multicolumn{2}{|c|}{ 95\% Confidence Interval } & \multirow{2}{*}{ P-value } \\
\hline & $<10$ days & $\geq 10$ days & & Lower & Upper & \\
\hline Nausea & $11.0 \%$ & $13.9 \%$ & 1.307 & 0.760 & 2.249 & 0.332 \\
\hline Vomiting & $7.2 \%$ & $8.0 \%$ & 1.12 & 0.569 & 2.204 & 0.742 \\
\hline Diarrhea & $12.3 \%$ & $12.4 \%$ & 1.008 & 0.591 & 1.717 & 0.000 \\
\hline Nausea and Vomiting & $12.0 \%$ & $15.0 \%$ & 1.291 & 0.764 & 2.182 & 0.043 \\
\hline Elevated INR (>1.5) & $13.1 \%$ & $11.1 \%$ & 0.827 & 0.431 & 1.586 & 0.567 \\
\hline Elevated Transaminases (AST > 40 and ALT > 45) & $64.3 \%$ & $62.2 \%$ & 0.915 & 0.633 & 1.323 & 0.637 \\
\hline AST > 40 only & $61.5 \%$ & $61.2 \%$ & 0.989 & 0.686 & 1.424 & 0.952 \\
\hline ALT $>45$ only & $30.2 \%$ & $29.2 \%$ & 0.951 & 0.644 & 1.403 & 0.799 \\
\hline Low Albumin $(<3.5)$ & $39.7 \%$ & $45.6 \%$ & 1.275 & 0.887 & 1.832 & 0.188 \\
\hline
\end{tabular}

TABLE 4: Predictor of prolonged hospital stay ( $\geq 10$ days)

INR: International normalized ratio; AST: Aspartate transaminase; ALT: Alanine transaminase.

Patients with elevated ALT only (OR = 1.594, $\mathrm{p}=0.019)$, AST only $(\mathrm{OR}=2.149, \mathrm{p}<0.001)$, and both ALT and AST $(\mathrm{OR}=2.022, \mathrm{p}<0.001)$ were all significant predictors of intensive care unit $(\mathrm{ICU}) /$ progressive care unit 
(PCU) care (Table 5). Symptoms including nausea, vomiting, and diarrhea did not lead to increased odds of ICU/PCU care (Table 5).

\begin{tabular}{|c|c|c|c|c|c|}
\hline \multirow{2}{*}{ Symptoms } & \multirow{2}{*}{$\begin{array}{l}\text { ICU/PCU Care } \\
\text { Admission rates }\end{array}$} & \multirow{2}{*}{ OR } & \multicolumn{2}{|c|}{ 95\% Confidence Interval } & \multirow{2}{*}{ P-value } \\
\hline & & & Lower & Upper & \\
\hline Nausea & $10.1 \%$ & 0.707 & 0.396 & 1.262 & 0.239 \\
\hline Vomiting & $5.9 \%$ & 0.712 & 0.34 & 1.491 & 0.366 \\
\hline Diarrhea & $10.9 \%$ & 0.827 & 0.475 & 1.442 & 0.504 \\
\hline Nausea and Vomiting & $14.3 \%$ & 0.761 & 0.435 & 1.331 & 0.337 \\
\hline Elevated INR (>1.5) & $13.6 \%$ & 1.206 & 0.636 & 2.286 & 0.566 \\
\hline Elevated Transaminases (AST > 40 and ALT > 45) & $72.6 \%$ & 2.022 & 1.37 & 2.986 & 0.000 \\
\hline AST $>40$ only & $71.6 \%$ & 2.149 & 1.461 & 3.161 & 0.000 \\
\hline ALT > 45 only & $36.0 \%$ & 1.594 & 1.078 & 2.357 & 0.019 \\
\hline Low Albumin $(<3.5)$ & $46.2 \%$ & 1.318 & 0.913 & 1.904 & 0.140 \\
\hline
\end{tabular}

TABLE 5: Predictor for requirement of ICU/PCU care

ICU: Intensive care unit; PCU: Progressive care unit; INR: International normalized ratio; AST: Aspartate transaminase; ALT: Alanine transaminase.

\section{Discussion}

We conducted a retrospective review of patients admitted with gastrointestinal manifestations following COVID-19 infection. Gastrointestinal involvement was assessed by reviewing gastrointestinal symptoms, and liver function test abnormalities. Our findings demonstrate longer hospital stays ( $\geqslant 10$ days) among patients manifesting with gastrointestinal symptoms including diarrhea, and a combination of nausea and vomiting. However, we also found that the presence of gastrointestinal symptoms was suggestive of a reduced 30-day mortality rate. Hepatic predictors including elevated ALT and AST had a strong association with the 30-day mortality rate and critical care requirement. Low albumin and elevated INR did not have any impact on the prognosis of patients with COVID-19 infection in our cohort.

The underlying mechanism for gastrointestinal involvement in COVID-19 is similar to that of respiratory and due to the attachment of the SARS-CoV-2 virus to the angiotensin-converting enzyme 2 (ACE-2) receptor in the digestive system, particularly the proximal large intestine [5]. Previous studies have reported data on the involvement of the gastrointestinal tract and liver [6,7]. Mao et al. identified a pooled prevalence of $15 \%$ for gastrointestinal symptoms in COVID-19 of whom 10\% did not have respiratory involvement [2]. Gastrointestinal symptoms have been observed among patients with COVID-19 infection in literature with discrepancies in their predictive prognostic potential $[8,9]$. In our cohort, GI symptoms had an inverse protective relationship with the 30-day mortality rate. However, our analysis demonstrates a significant impact of diarrhea, and nausea, and vomiting in combination on the duration of hospital stay. Additionally, the gastrointestinal manifestations had no contribution to the patients' requirements for critical care admissions. With conflicting reports of the impact of gastrointestinal symptoms on COVID-19 infection outcomes, our analysis also demonstrates both their positive and negative prognostic potential.

Our findings suggest that, as the liver involvement is suggested through ALT and AST elevation, patients have worse 30-day mortality rates and require critical care with the difference proving significant. Reports of liver injury have been suggested earlier including elevated transferases and INR, and decreased albumin levels, among patients with COVID-19 [10,11]. While our findings did not identify low serum albumin as a prognostic marker as reported by Huang et al. [12], elevated transaminases were suggestive of worse outcomes in our cohort of COVID-19-infected patients. Our findings from recent literature show that elevated AST and/or ALT are associated with increased mortality rates.

\section{Strengths and limitations}

The main strengths of our study include a large sample size $(n=520)$ and the longitudinal study design in New York City, a prominent epicenter of the COVID-19 pandemic. The lab parameters and gastrointestinal manifestations were assessed on the day of admission. To the best of our knowledge, this is the first study that observes both liver function tests as well as gastrointestinal symptoms on the day of hospital admission to assess their prognostic potential. 
The main limitations of our study include the lack of categorization of the existing burden of chronic liver disease and the use of medications that might be hepatotoxic. As the gastrointestinal symptoms and liver injury markers were assessed at the time of hospital admission, patients with pre-existing liver injury may have been included. No testing was conducted to assess the hepatitis B and C status of these patients. Similarly, the baseline comorbidities and other health parameters, i.e. presence of respiratory symptoms, were not controlled for in the analysis.

\section{Recommendations}

Gastrointestinal manifestations of COVID-19 may manifest early in the course of the disease, possibly occurring in isolation. Management of patients with COVID-19 infection should also contain the potential prognostic impact of liver markers and gastrointestinal symptoms. With a great interest in the prognostic capacity of gastrointestinal symptoms and liver involvement, this study identifies a worse prognosis among COVID-19 patients. While the main target organ of COVID-19 infection remains the respiratory system, the impact of gastrointestinal involvement may not be undermined as has been observed in the literature [1315].

\section{Conclusions}

Elevated liver transaminases including ALT and AST at the time of hospital admission can predict critical care requirement and all-cause 30-day hospital mortality in patients with COVID-19 infection. Presence of gastrointestinal symptoms is associated with worsened outcomes.

\section{Additional Information \\ Disclosures}

Human subjects: Consent was obtained or waived by all participants in this study. Animal subjects: All authors have confirmed that this study did not involve animal subjects or tissue. Conflicts of interest: In compliance with the ICMJE uniform disclosure form, all authors declare the following: Payment/services info: All authors have declared that no financial support was received from any organization for the submitted work. Financial relationships: All authors have declared that they have no financial relationships at present or within the previous three years with any organizations that might have an interest in the submitted work. Other relationships: All authors have declared that there are no other relationships or activities that could appear to have influenced the submitted work.

\section{Acknowledgements}

Syed Hashim Abbas Ali Bokhari; Aga Khan University, Karachi, Pakistan; Role: Data analysis Donald Hathaway; Medical Student IV- St Georges University, Graneda; Role: Data collection Shayan Zaheer; Medical Student IV- St Georges University Grenada; Role: Data collection Brian Altonen MPH; Woodhull Hospital, Brooklyn, NY; Role: Data Analysis

\section{References}

1. Guan WJ, Ni ZY, Hu Y, et al.: Clinical characteristics of coronavirus disease 2019 in China . N Engl J Med. 2020, 382:1708-1720. 10.1056/NEJMoa2002032

2. Mao R, Qiu Y, He JS, et al.: Manifestations and prognosis of gastrointestinal and liver involvement in patients with COVID-19: a systematic review and meta-analysis. Lancet Gastroenterol Hepatol. 2020, 5:667678. 10.1016/S2468-1253(20)30126-6

3. Pirola CJ, Sookoian S: COVID-19 and ACE2 in the liver and gastrointestinal tract: putative biological explanations of sexual dimorphism. Gastroenterology. 2020, 159:1620-1621. 10.1053/j.gastro.2020.04.050

4. Zhong P, Xu J, Yang D, et al.: COVID-19-associated gastrointestinal and liver injury: clinical features and potential mechanisms. Signal Transduct Target Ther. 2020, 5:256. 10.1038/s41392-020-00373-7

5. Xiao F, Tang M, Zheng X, Liu Y, Li X, Shan H: Evidence for gastrointestinal infection of SARS-CoV-2. Gastroenterology. 2020, 158:1831-1833. 10.1053/j.gastro.2020.02.055

6. Beattie RM, Ashton JJ, Penman ID: COVID-19 and the gastrointestinal tract: recent data. Frontline Gastroenterol. 2020, 11:371-374. 10.1136/flgastro-2020-101602

7. Tian Y, Rong L, Nian W, He Y: Review article: gastrointestinal features in COVID-19 and the possibility of faecal transmission. Aliment Pharmacol Ther. 2020, 51:843-851. 10.1111/apt.15731

8. Ramachandran P, Onukogu I, Ghanta S, Gajendran M, Perisetti A, Goyal H, Aggarwal A: Gastrointestinal symptoms and outcomes in hospitalized coronavirus disease 2019 patients. Dig Dis. 2020, 38:373-379. 10.1159/000509774

9. Buscarini E, Manfredi G, Brambilla G, et al.: GI symptoms as early signs of COVID-19 in hospitalised Italian patients. Gut. 2020, 69:1547-1548. 10.1136/gutjnl-2020-321434

10. Pozzobon FM, Perazzo H, Bozza FA, Rodrigues RS, de Mello Perez R, Chindamo MC: Liver injury predicts overall mortality in severe COVID-19: a prospective multicenter study in Brazil (PREPRINT). Hepatol Int. 2021, 10.1007/s12072-021-10141-6

11. Bahardoust M, Heiat M, Khodabandeh M, et al.: Predictors for the severe coronavirus disease 2019 (COVID19) infection in patients with underlying liver disease: a retrospective analytical study in Iran. Sci Rep. 2021, 11:3066. 10.1038/s41598-021-82721-3

12. Huang J, Cheng A, Kumar R, Fang Y, Chen G, Zhu Y, Lin S: Hypoalbuminemia predicts the outcome of 


\section{Cureus}

COVID-19 independent of age and co-morbidity. J Med Virol. 2020, 92:2152-2158. 10.1002/jmv.26003

13. Tariq R, Saha S, Furqan F, Hassett L, Pardi D, Khanna S: Prevalence and mortality of COVID-19 patients with gastrointestinal symptoms: a systematic review and meta-analysis. Mayo Clin Proc. 2020, 95:16321648. 10.1016/j.mayocp.2020.06.003

14. Aguila EJT, Cua IHY, Fontanilla JAC, Yabut VLM, Causing MFP: Gastrointestinal manifestations of COVID19: impact on nutrition practices. Nutr Clin Pract. 2020, 35:800-805. 10.1002/ncp.10554

15. Agarwal A, Chen A, Ravindran N, To C, Thuluvath PJ: Gastrointestinal and liver manifestations of COVID19. J Clin Exp Hepatol. 2020, 10:263-265.10.1016/j.jceh.2020.03.001 